\title{
Light-regulated Molecular Trafficking in a Synthetic Water-soluble Host
}

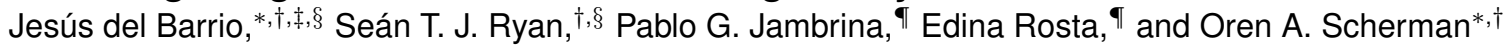 \\ Melville Laboratory for Polymer Synthesis, Department of Chemistry, University of Cambridge, Cambridge \\ CB2 1EW, U.K., Schlumberger Gould Research, Madingley Road, Cambridge, CB3 OEL, U.K., and \\ Department of Chemistry, King's College London, London SE1 1DB, U.K. \\ Received February 13, 2016; E-mail: JBarrio2@slb.com; oas23@cam.ac.uk
}

\begin{abstract}
The cucurbit[8]uril (CB[8])-mediated complexation of a dicationic azobenzene in water allows for the light-controlled encapsulation of a variety of second guest compounds, including amino acids, dyes and fragrance molecules. Such controlled guest sequestration inside the cavity of $\mathrm{CB}[8]$ enables the regulation of the thermally induced phase transition of poly ( $N$-isopropylacrylamide) - which is not photosensitive - thus demonstrating the robustness and relevancy of the light-regulated $\mathrm{CB}[8]$ complexation.
\end{abstract}

Light energy transduction is a fundamental phenomenon in the biosphere where living organisms are able to exploit sophisticated noncovalent constructs to transform light into kinetic and potential energy. For instance, visual transduction is initiated by the photoisomerization of retinal in the integral membrane protein Bacteriorhodopsin (BR), and results in the release of a proton to the extracellular environment. ${ }^{1}$ It is widely accepted that the exquisite control over the light-induced proton transfer is largely imparted by the delicate organization of the protein binding site. At a lower level of complexity, synthetic chemistry has enabled the production of artificial systems that, similarly to BR, can also bind and release protons and substantially larger species through a diversity of light-controlled processes. ${ }^{2}$ A variety of examples exist, including molecular tweezers, foldamers, cages and macrocycles. ${ }^{3}$ However, the design and synthesis of light-regulated containers that can operate in an aqueous environment remains a challenge, with only a few examples related to modified cyclodextrins. ${ }^{4}$ Such materials, provided they show reversible and tight binding to both neutral and charged species, could have significant impact in a variety of areas including photopharmacotherapy, drug delivery and encapsulation technologies.

Here we describe a supramolecular container system exhibiting light-controlled encapsulation properties in water. Our multicomponent approach towards photoresponsive binding relies on an optimized design of its individual constituents and comprises the barrel-shaped molecule cucurbit[8]uril $(\mathrm{CB}[8])^{5}$ and a photoresponsive ancillary guest (Chart 1). Recently, we reported the controlled complexation of neutral guests in $\mathrm{CB}[8]$ aided by a series of aromatic bis(imidazolium) derivatives. ${ }^{6}$ Depending on their size, these salts can act as first guests for $\mathrm{CB}[8]$ enabling the encapsulation of relatively small second guests includ-

\footnotetext{
${ }^{\dagger}$ Melville Laboratory for Polymer Synthesis, Department of Chemistry, University of Cambridge, Cambridge CB2 1EW, U.K.

${ }^{\ddagger}$ Schlumberger Gould Research, Madingley Road, Cambridge, CB3 OEL, U.K.

`Department of Chemistry, King's College London, London SE1 1DB, U.K.

$\S$ These authors contributed equally
}

ing acetone, acetonitrile and diethyl ether. The $E$ isomers of azobenzenes $\mathbf{1}$ and $\mathbf{2}$ are also extended organic structures flanked by positively charged groups (Chart $1 \mathrm{~b}$ and Scheme 1). We therefore examine these molecules as responsive first guests that can potentially allow for the photochemical manipulation of the binding properties of $\mathrm{CB}[8]$ complexes.

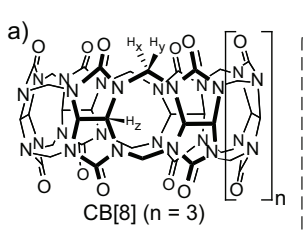

c)

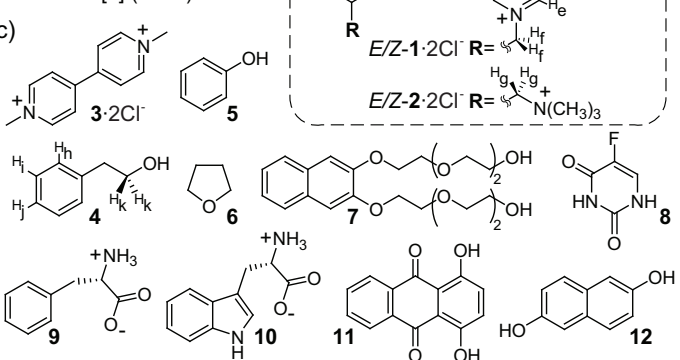

Chart 1. Structures of $\mathrm{CB}[8]$ (a), photoresponsive first guests molecules 1 and 2 (b) and selected guests 3-12 (c).

The binding of azobenzene $E-\mathbf{1}$ to $\mathrm{CB}[8]$ was initially investigated by electronic absorbance spectroscopy (Figure S1). The main absorption band of $E$-1 shifts to shorter wavelengths when the azobenzene guest is added to an aqueous solution of $\mathrm{CB}[8]$, whereas the $n-\pi^{*}$ band redshifts and its intensity increases compared to free $E$-1. The structure of the complex prepared from equimolar amounts of $E-\mathbf{1}$ and $\mathrm{CB}[8]$ was further investigated by NMR spectroscopy. The ${ }^{1} \mathrm{H}$ NMR spectrum of $E-\mathbf{1}$ showed five distinct aromatic proton signals in the 7.60-9.10 ppm region (Figure 1a). However, in the presence of $\mathrm{CB}[8]$, the symmetry of $E-\mathbf{1}$ is reduced and the bound azobenzene exhibits up to ten sharp, well-defined, and widely dispersed aromatic proton signals (Figure 1b). Compared to free $E-\mathbf{1}$, the signals corresponding to the protons of one of the pyridinium groups of $E-\mathbf{1}$ $\left(\mathrm{H} c^{*}-e^{*}\right.$ in Figure $\left.1 \mathrm{~b}\right)$ significantly shift upfield in the presence of $\mathrm{CB}[8]$, suggesting that this part of the molecule lays deep inside the cavity of the host. In contrast, the other pyridinium group is positioned at the ureidyl carbonyl portal area of $\mathrm{CB}[8]$ according to the characteristic downfield shift of some of its proton resonances ( $\mathrm{H} c$ and $\mathrm{H} d$ in Figure $1 \mathrm{~b}$ ). A series of ${ }^{1} \mathrm{H}$ diffusion ordered (DOSY) NMR experiments were performed in order to investigate the size of this complex (Figures 1 and S6). These experiments revealed that the diffusion coefficient $(D)$ of the complex resulting from an equimolar mixture of $E-\mathbf{1}$ and $\mathrm{CB}[8]$ is ca. $79 \%$ of that for the binary complex of the model guest 1,1'-dimethyl-4,4'- 
bipyridinium dication (3 in Chart 1) and $\mathrm{CB}[8]$ (Figure S5). This means that the CB[8] complex of $E-\mathbf{1}$ is approximately twice as large as $\mathrm{CB}[8] \cdot 3$ (Figure S6). Considering its size, its binding stoichiometry (Figure S2) and the fact that the cavity of $\mathrm{CB}[8]$ is large enough to accommodate up to two flat aromatic moieties, we formulate the structure resulting from a 1:1 mixture of $\mathrm{CB}[8]$ and $E-1$ as a four-component $\mathrm{CB}[8]_{2} \cdot E-\mathbf{1}_{2}$ complex (Scheme 1a and Figure S25).

Scheme 1. Formation of host-guest complexes of $\mathrm{CB}[8]$ with 1 (a), and stepwise formation of 1:1:1 ternary complex of $\mathrm{CB}[8]$ with $E-2$ and second guest molecule followed by light induced release $(b)$.

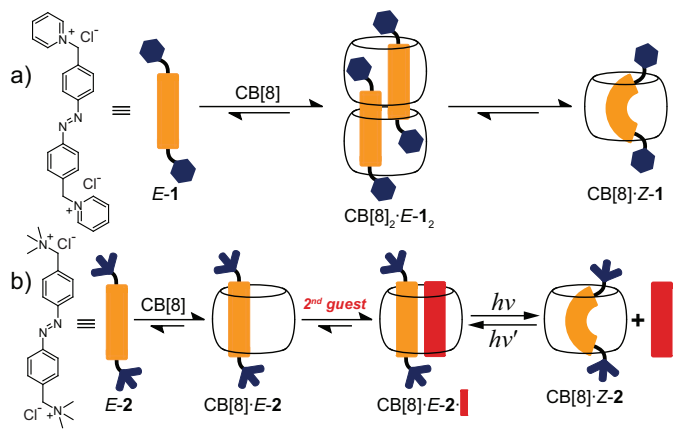

DFT calculations (M062X/6-31G* level of theory) showed that $E \mathbf{- 1}$ dimerization in $\mathrm{CB}[8]_{2} \cdot E-\mathbf{1}_{2}$ is a favorable process compared to a purely bimolecular association of $\mathrm{CB}[8]$ and E-1 (Figure S30). Furthermore, the calculated structure of the quaternary complex is in full agreement with the asymmetrical encapsulation of $E-\mathbf{1}$, as suggested by NMR data (vide supra), and shows that one pyridinium group of each $E-1$ is accommodated inside the cavity of $\mathrm{CB}[8]$ while the other resides at the portal (See Figure S25).

When an aqueous solution of $\mathrm{CB}[8]_{2} \cdot E-\mathbf{1}_{2}$ is stored at $298 \mathrm{~K}$ in the absence of light, a new set of proton NMR signals - incompatible with the 2:2 complex - arises (Figures 1c and S15). The resonances corresponding to protons $\mathrm{H} a$ and $\mathrm{H} b$ of 1 show up as doublets at 5.90 and $6.82 \mathrm{ppm}$, indicating that the azobenzene is still encapsulated inside the cavity of $\mathrm{CB}[8]$. In contrast, the pyridinium groups may reside at the ureidyl carbonyl portal area according to the shifting of their proton resonances (signals in the 8.10-9.05 ppm region, Figure 1c). The new species possesses a smaller size than $\mathrm{CB}[8]_{2} \cdot E-\mathbf{1}_{2}$, as evidenced by its larger $D$ value (Figures 1 and S7). Major changes were also observed in the electronic absorbance spectrum of $\mathrm{CB}[8]_{2} \cdot E-\mathbf{1}_{2}$ simultaneously to the evolution of the ${ }^{1} \mathrm{H}$ NMR spectrum (vide supra). The intensity of the main electronic absorbance band decreased with time, which is typically ascribed to a decrease of the $E: Z$ ratio. ${ }^{7}$ In combination, these results suggest that $\mathrm{CB}[8]$ promotes the thermal $E \rightarrow Z$ isomerization of $\mathbf{1}$, with tetramer $\mathrm{CB}[8]_{2} \cdot E-\mathbf{1}_{2}$ as a metastable intermediate of the process (Scheme 1a). The formation of $\mathrm{CB}[8] \cdot Z-1$ from an equimolar mixture of $\mathrm{CB}[8]$ and $E-1$, which can be accelerated by UV light irradiation (Figure S1), overcompensates the thermodynamic cost associated with the break-up of the $\mathrm{CB}[8]_{2} \cdot E-\mathbf{1}_{2}$ homodimer and the thermal $E \rightarrow Z$ isomerization of 1 .

Electronic structure calculations predicted that azobenzene $E$-2 (Chart 1b), with flanking trimethylammonim groups, also binds to $\mathrm{CB}[8]$ in a $1: 1$ ratio and, in contrast to $E-\mathbf{1}$, forms a binary CB[8] $E-2$ complex (Figures 2 and S27). This was confirmed by the downfield shift of the signals corresponding to protons $\mathrm{H} a, \mathrm{H} b$ and $\mathrm{H} g$ (Figure S17). Also, a $D$ value of $(2.73 \pm 0.04) \times 10^{-10} \mathrm{~m}^{2} \mathrm{~s}^{-1}$ was obtained by ${ }^{1} \mathrm{H}$ DOSY NMR spectroscopy, which in combination to ITC results (Figure S2), suggests an absolute stoichiometry of 1:1 (Figure S8). A binding constant $K_{1}$, on the order of $10^{6} \mathrm{M}^{-1}$ was obtained by isothermal titration calorimetry (lTC, see Figure S2). A series of ${ }^{1} \mathrm{H}$ NMR experiments confirmed, at least in the timeframe of this study, that the $E$ isomer of $\mathbf{2}$ is the most thermodynamically stable form in the presence of $\mathrm{CB}[8]$ (Figure S16), likely on account of its bulkier $\mathrm{NMe}_{3}{ }^{+}$groups; therefore, it can partake in stable heteroternary complexation (Scheme 1b).

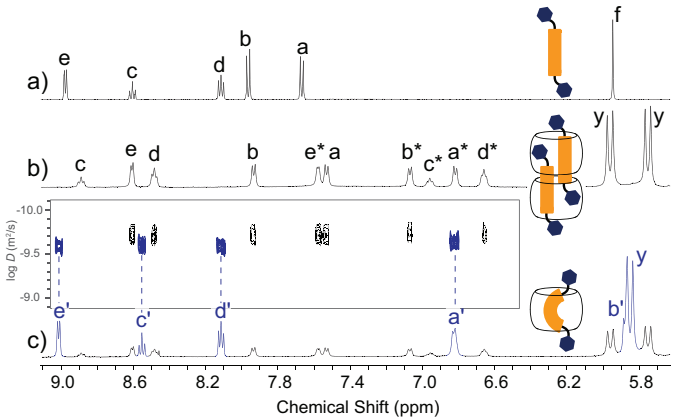

Figure 1. Partial ${ }^{1} \mathrm{H}$ NMR spectra (500 MHz, $\left.\mathrm{D}_{2} \mathrm{O}, 298 \mathrm{~K}\right)$ of $E-\mathbf{1}$ (a), a freshly prepared solution of an equimolar mixture of $\mathrm{CB}$ [8] and $E-\mathbf{1}$ (b) and after storage in the dark at $298 \mathrm{~K}$ for $\sim 3$ months (c) and corresponding ${ }^{1} \mathrm{H}$ DOSY plots. Signals corresponding to $Z \mathbf{- 1}$ protons in (c) are indicated with apostrophes. The two groups of ${ }^{1} \mathrm{H}$ NMR signals of the asymmetrically encapsulated $E$ $\mathbf{1}$ in (b) are denoted by $\mathrm{H} a-e$ and $\mathrm{H} a^{*}-e^{*}$ (see Chart 1 for proton labeling and text for more details).

To investigate the potential formation of ternary complexes, we selected 2-phenylethanol (4 in Chart 1c), a naturally-occurring alcohol and also a fragrance molecule, ${ }^{8}$ as a model second guest compound. The ${ }^{1} \mathrm{H}$ NMR spectrum of an equimolar mixture of $\mathrm{CB}[8], E-\mathbf{2}$ and $\mathbf{4}$ showed an upfield shift perturbation and substantial broadening of the aromatic signals of the alcohol upon binding to $\mathrm{CB}[8] \cdot E-2$ (Figure 3). These changes are consistent with those previously observed for the $\mathrm{CB}[8] \cdot \mathbf{3}$ complexation of aromatic moieties such as 2,6-dihydroxynaphthalene (12 in Chart 1c). ${ }^{9}$ The formation of a $\mathrm{CB}[8] \cdot E-\mathbf{2} \cdot \mathbf{4}$ complex was further supported by ${ }^{1} \mathrm{H}$ DOSY NMR spectroscopy (Figure S10) and $a b$ initio calculations (Figures 2, S29 and S31). While azobenzenes $\mathbf{1}$ and $\mathbf{2}$ share many similar structural and physical characteristics, none of the complexes comprising $\mathrm{CB}[8]$ and $\mathbf{1}$, neither the transient $\mathrm{CB}[8]_{2} \cdot E-\mathbf{1}_{2}$ nor the most stable $\mathrm{CB}[8] \cdot Z \mathbf{- 1}$, allowed for the encapsulation of $\mathbf{4}$ as evidenced by ${ }^{1} \mathrm{H}$ NMR spectroscopy (Figure S18).

The binary complex $\mathrm{CB}[8] \cdot E-2$ can bind a variety of neutral extended aromatic guests, as well as more chemically and biologically relevant species including amphiphiles, dyes (quinizarin, $\mathbf{1 1}$ in Chart 1c, which is also a structural analogue to the antineoplastic agent mitoxantrone), the amino acids L-phenylalanine and L-tryptophan, and the antimetabolite 5-fluorouracil (guests 8-10 in Chart 1c). In addition to NMR characterization (Figures S11-S13), we have performed ITC experiments to obtain a full thermodynamic description of the binding of $\mathrm{CB}[8] \cdot E-2$ to a library of second guests (Figure S3 and Table S1). The second guest association constants, $K_{2}$, were in the range of $10^{3}-10^{5} \mathrm{M}^{-1}$. Association is governed by enthalpy, which is consistent with other $\mathrm{CB}[8]$ ternary complexes and likely related to the release of high energy water molecules from the $\mathrm{CB}[8] \cdot E-2$ cavity upon formation of the ternary complex. ${ }^{10}$ Furthermore, not only aromatic but also aliphatic guests, such as 
tetrahydrofuran (6 in Chart 1c), can be encapsulated within $\mathrm{CB}[8] \cdot E-2$.

The photoisomerization properties of $\mathbf{2}$ in the presence and absence of $\mathrm{CB}[8]$ were initially investigated by electronic absorbance spectroscopy. The intensity of the band corresponding to the $\pi-\pi^{*}$ transition of $E-2$ decreased upon UV light irradiation $(350 \mathrm{~nm})$, and two new bands appeared at $\sim 305$ and $\sim 425 \mathrm{~nm}$ (Figure S1). These bands were related to $Z \mathbf{- 2}$ and the changes in the spectrum were ascribed to a decrease of the $E: Z$ ratio upon UV light irradiation. ${ }^{7}$ When an aqueous solution of $\mathrm{CB}[8] \cdot E-2$ was exposed to UV light, the intensity of the main band also decreased, whereas the $\mathrm{n}-\pi^{*}$ became weaker and blue shifted. These observations are comparable to those described for analogous cationic azobenzene guests in the presence of $\mathrm{CB}[8]$ and we were therefore led to believe that the interaction of $Z-2$ and $C B[8]$ may be similar to that reported previously, ${ }^{11}$ with $Z-2$ included inside the cavity of the macrocycle. ${ }^{12}$ This was supported by the upfield shift of the signals of protons $\mathrm{H} a, \mathrm{H} b$ and $\mathrm{H} g$, relative to those of free $Z-2$ (Figure S17), and the fact that $Z-2$ and $\mathrm{CB}[8]$ still share a single $D$ value after UV light irradiation as measured by ${ }^{1} \mathrm{H}$ DOSY NMR (Figure S9).
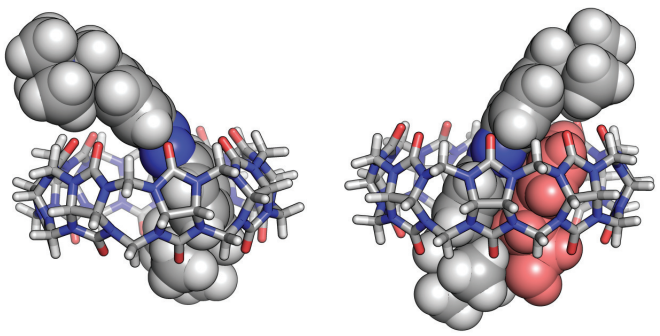

Figure 2. Geometry-optimized molecular structures (M062X/6$31 \mathrm{G}^{*}$ level of theory) of $\mathrm{CB}[8] \cdot E-2$ (left) and $\mathrm{CB}[8] \cdot E-\mathbf{2} \cdot 4$ (right) complexes.

The exposure of an aqueous solution of the ternary complex, $\mathrm{CB}[8] \cdot E-\mathbf{2} \cdot \mathbf{4}$, to UV light induces the $E \rightarrow Z$ isomerization of $\mathbf{2}$ and the release of $\mathbf{4}$ from the multicomponent container (Scheme 1b). This was revealed by the upfield shift of the signals of protons $\mathrm{H} a, \mathrm{H} b$ and $\mathrm{H} g$ of 2 and the downfield shift of the aromatic proton resonances of 4 (Figure 3). Also, ${ }^{1} \mathrm{H}$ DOSY NMR analysis showed that $\mathrm{CB}[8] \cdot Z$ $\mathbf{2}$ and $\mathbf{4}$ diffuse as independent molecular species after UV light irradiation (Figure S14). Although quantitative conversion of $E-2$ into $Z-2$ and concomitant release of 4 cannot be attained after UV light irradiation, the system, at equilibrium, reaches a highly $\mathrm{CB}[8] \cdot Z-2$ enriched state $(E: Z$ ratio $\sim 1: 5$ ) with the bimolecular complex and free 4 as the major components. Irradiation of the mixture with visible light $(420 \mathrm{~nm})$, reverts the system back to the $\mathrm{CB}[8] \cdot \boldsymbol{E}-\mathbf{2} \cdot \mathbf{4}$ enriched state. The system can be reversibly cycled many times between the $E-\mathbf{2}$ and the $Z$-2 enriched states by alternating exposure to UV and visible light (Figure S20). An analogous photoregulated phenomena was also observed for the ternary complexes involving $\mathrm{CB}[8] \cdot E-2$ and the previously mentioned library of second guest molecules.

The underlying basis for the photocontrolled "catch-andrelease" process is that $Z \mathbf{- 2}$ fully occupies the cavity of $\mathrm{CB}[8]$ with a binding constant much higher than that of the second guests. A series of ITC experiments allowed us to confirm our hypothesis. The $E$ isomer of $\mathbf{2}$ exhibits a binding constant on the order of $10^{6} \mathrm{M}^{-1}$. After UV light irradiation, the decrease of the $E: Z$ ratio is accompanied by an increase of one order of magnitude in $K_{a}$. In comparison, second guest molecules partake in heteroternary $\mathrm{CB}[8]$ complex formation with much lower $K_{2}$, typically on the order of $10^{3}-10^{5} \mathrm{M}^{-1} .^{5}$ The high affinity of $Z-2$ towards CB[8] is likely on account of the increased volume the molecule occupies (Table S2), in contrast to the more extended $E$-2, and its ability to fully occupy the cavity of $\mathrm{CB}[8]$. Moreover, iondipole interactions between the electron-poor $\mathrm{NMe}_{3}{ }^{+}$groups and the electron-rich ureidyl carbonyl portals of $\mathrm{CB}[8]$ are favored in $\mathrm{CB}[8] \cdot Z-2$ as the distance between the $\mathrm{NMe}_{3}{ }^{+}$ groups decreases from $\sim 13 \AA$, in $E$-2, to $\sim 7 \AA$ in $Z$-2.

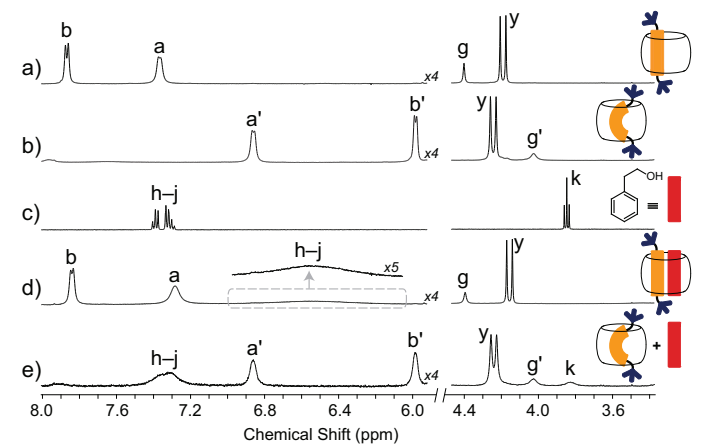

Figure 3. Selected regions of the ${ }^{1} \mathrm{H}$ NMR spectra $(500 \mathrm{MHz}$, $\left.\mathrm{D}_{2} \mathrm{O}, 298 \mathrm{~K}\right)$ of $(\mathrm{a}, \mathrm{b}) \mathrm{CB}[8] \cdot 2$ before (a) and after (b) UV light irradiation, 4 (c), and (d, e) CB[8] $E$-2.4 before (d) and after (e) UV light irradiation. See Chart 1 for proton labeling. Signals corresponding to protons of $Z \mathbf{- 2}$ are indicated with apostrophes.

The ability of $\mathrm{CB}[8] \cdot E-2$ to catch and release guests in a remote fashion led us to consider whether lightcontrolled complexation could also govern transformations, which are not known to be photoresponsive. ${ }^{13}$ We anticipated that it could serve, for example, as an external handle on the thermally induced phase transition of poly( $N$-isopropylacrylamide) (PNIPAM). In an effort to evaluate this idea, the random terpolymer $\mathbf{P 1}$ $\left(M_{n}: 55 \mathrm{kDa}, \mathrm{PDI}: 1.25\right.$, Figure 4) was prepared by statistical copolymerization of $N$-isopropylacrylamide and $N$ (2-hydroxyethyl)acrylamide and subsequent modification of a fraction of the hydroxyl groups with 2-(4-biphenyl)ethyl isocyanate (Scheme $\mathrm{S} 2) .{ }^{14}$

In the presence of $\mathrm{CB}[8] \cdot E-\mathbf{2}$, the structural design of $\mathbf{P 1}$ allows for the formation of ternary complexes between the pendant biphenyl units of the polymer, E-2 and CB[8] (Figure $4 \mathrm{a})$. This was shown through a combination of ${ }^{1} \mathrm{H}$ NMR spectroscopy and ITC experiments (Figure S3). With regard to the thermoresponsive properties of $\mathbf{P 1}$, the presence of pendant biphenyl and hydroxyl groups has a large influence in the polymer cloud-point temperature $\left(T_{c p}\right)$. An aqueous solution of P1 (0.1 mM) exhibits a $T_{c p}$ of $43.0{ }^{\circ} \mathrm{C}$ (Figure 4b), a temperature that is higher than that of unmodified PNIPAM $\left(32.6{ }^{\circ} \mathrm{C}\right)$. This difference in $T_{c p}$ is a result of the larger content of hydrophilic $N$-(2-hydroxyethyl)acrylamide repeating units compared to the hydrophobic biphenyl group content. Upon addition of one equivalent of $\mathrm{CB}[8] \cdot E-\mathbf{2}$, the $T_{c p}$ of the polymer system increases to $49.5^{\circ} \mathrm{C}$ (Figure $4 \mathrm{~b}$ ). This change can be attributed to the effect of side-chain complexation, which results in a switch from the hydrophobic biphenyl moieties to the hydrophilic doubly-charged ternary complexes. After UV light irradiation, the $T_{c p}$ of the polymer system drops to $45.4{ }^{\circ} \mathrm{C}$ as the $\mathrm{CB}[8] \cdot Z$-2 complexes are released from $\mathbf{P} \mathbf{1}$ and the hydrophobic biphenyl groups are unmasked (Figure $4 \mathrm{~b})$. The original $T_{c p}$ of $\mathbf{P 1}\left(43.0^{\circ} \mathrm{C}\right)$ cannot be fully recovered due to the inherent $E: Z$ composition of the photostationary state of $\mathrm{CB}[8] \cdot 2$ after UV light 
irradiation.

The light-controlled switching of the $T_{c p}$ enabled us to reversibly transition between a clear and a turbid solution of $\mathbf{P 1}, \mathbf{2}$ and $\mathrm{CB}[8]$ at a constant temperature. At $47{ }^{\circ} \mathrm{C}$, a mixture of $\mathbf{P 1}$ and $\mathrm{CB}[8] \cdot E-\mathbf{2}$ (1 equivalent of the binary complex per biphenyl group) in water is clear, as illustrated in Figure 4b. After UV light irradiation at the same temperature, the solution turns turbid as a result of the decomplexation of the biphenyl groups of the polymer (the mixture of $\mathrm{CB}[8], \mathbf{2}$ and $\mathbf{P 1}$ after UV light exposure has a $T_{c p}$ of $45.4^{\circ} \mathrm{C}$, which is lower than $47^{\circ} \mathrm{C}$ ). Irradiation with visible light induces an increase in the transmittance of the solution, from turbid back to clear again, and many cycles of alternating turbid/clear states can be carried out by using light of the appropriate frequency (Figure 4c).
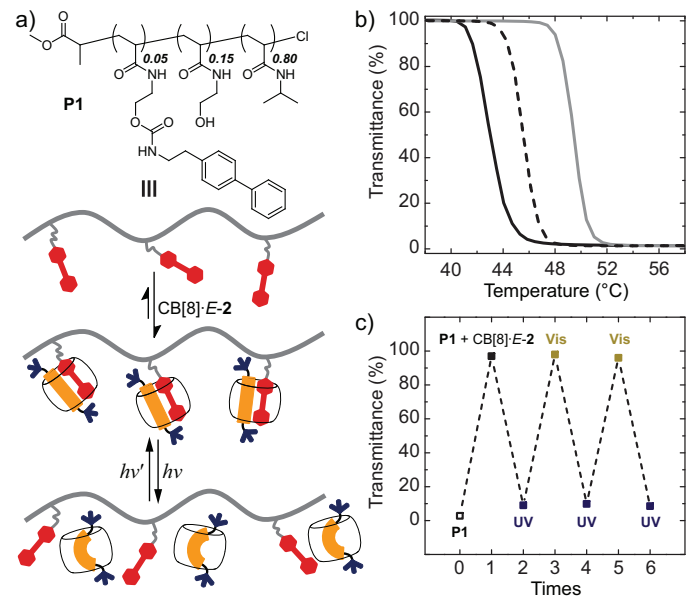

Figure 4. Structure of $\mathbf{P 1}$ and schematic representation of the light-controlled complexation of the biphenyl moieties of $\mathbf{P 1}$ (a). Transmittance (recorded at $600 \mathrm{~nm}$ and at a heating rate of $\left.1{ }^{\circ} \mathrm{C} / \mathrm{min}\right)$ of $\mathbf{P 1}\left(0.1 \mathrm{mM}\right.$ in $\left.\mathrm{H}_{2} \mathrm{O}\right)$ as a function of temperature (b) in the absence (full black line) and presence (full gray line) of $\mathrm{CB}[8] \cdot E-2$, and after UV light irradiation (dashed line). Changes in the transmittance of $\mathbf{P 1}\left(0.1 \mathrm{mM}\right.$ in $\left.\mathrm{H}_{2} \mathrm{O}\right)$ at $47^{\circ} \mathrm{C}$ by alternating irradiation with UV and visible light in the presence of $\mathrm{CB}[8]$ and 2 (c).

In conclusion, we have shown that photochromic hostguest complexes, which quantitatively self-assemble from equimolar amounts of $\mathrm{CB}[8]$ and azobenzenes $E-\mathbf{1}$ and $E-\mathbf{2}$, exhibit an absolute stoichiometry imposed by the positivelycharged groups of the guest. The asymmetrical encapsulation of $E-1$ inside a $2: 2 \mathrm{CB}[8]$ complex exhibits a remarkable level of regioselectivity, considering the high symmetry of the reagents, and may enable the investigation of well-defined dye aggregates in solution. ${ }^{15}$ Although the complete elucidation of the energy landscape associated with the $\mathrm{CB}[8]$ complexes of azobenzene $\mathbf{1}$ is still a matter of further research in our laboratory, it is evident that neither $\mathrm{CB}[8]_{2} \cdot E$ $\mathbf{1}_{2}$ nor CB[8]·Z-1 allow for the formation of heteroternary complexes. Conversely, azobenzene $E-2$ binds to $\mathrm{CB}[8]$ in a 1:1 ratio to form a $\mathrm{CB}[8] \cdot E-2$ complex, thus allowing for the photocontrolled catch and release of a variety of second guest molecules. Equipping CB[8] with a photoresponsive ancillary guest may be regarded as a supramolecular approach to imparting photoresponsive properties to an otherwise photoinactive host, such that the complicated synthetic, covalent modification of host systems may be avoided. Our CB[8] catch-and-release strategy is a general approach to regulate the encapsulation of biologically important molecules, which may find applications in the fragrance industry ${ }^{16}$ and other fields including photopharmacology ${ }^{17}$ and optogenetics. ${ }^{18}$
Furthermore, it may become useful for the development of artificial molecular devices which, in a fashion reminiscent to BR, can perform complex light-activated functions. Finally, we have demonstrated that it can be readily exploited in systems with higher levels of complexity to regulate phenomena that are not known to be photoresponsive, such as solution phase transitions of thermoresponsive polymers.

Acknowledgement J.D.B. thanks a Marie Curie IEF (project no. 273807). S.T.J.R. thanks the Cambridge Home and European Scholarship Scheme and the Robert Gardiner memorial scholarship. This work was supported by the EPSRC (reference no. EP/G060649/1), an ERC Starting Investigator Grant (project no. 240629) and a Next Generation Fellowship from the Walters-Kundert Foundation. The authors thank HECBioSim via the ARCHER UK National Supercomputing Service, the NIH Biowulf cluster, and the Ada King's $\mathrm{HPC}^{3}$ service.

Supporting Information Available: This material is available free of charge via the Internet at http://pubs .acs . org/.

\section{References}

(1) (a) Zimmer, M. Chem. Rev. 2002, 102, 759-782; (b) Lanyi, J. K. Annu. Rev. Physiol. 2004, 66, 665-688.

2) (a) Balzani, V.; Credi, A.; Venturi, M. Chem. Eur. J. 2008 14, 26-39; (b) Göstl, R.; Senf, A.; Hecht, S. Chem. Soc. Rev. 2014, 43, 1982-1996.

(3) (a) Blank, M.; Soo, L. M.; Wassermann, H.; Erlanger, B. Science 1981, 214, 70-72; (b) Shinkai, S.; Ishihara, M.; Ueda, K. Manabe, O. J. Chem. Soc., Chem. Commun. 1984, 727-729; (c) Irie, M. Kato, M. J. Am. Chem Soc 1985, 107, 10241028; (d) Hua, Y.; Flood, A. H. J. Am. Chem. Soc 2010, 132, 12838-12840; (e) Han, M.; Michel, R.; He, B.; Chen, Y.-S.; Stalke, D.; John, M.; Clever, G. H. Angew. Chem., Int. Ed. 2013, 52, 1319-1323; (f) Dube, H.; Rebek, J. Angew. Chem., Int. Ed. 2012, 51, 3207-3210; (g) Clever, G. H.; Tashiro, S.; Shionoya, M. J. Am. Chem. Soc. 2010, 132, 9973-9975; (h) Muraoka, T.; Kinbara, K.; Aida, T. Nature 2006, 440, 512515.

(4) (a) Ueno, A.; Yoshimura, H.; Saka, R.; Osa, T. J. Am Chem. Soc. 1979, 101, 2779-2780; (b) Ueno, A.; Tomita, Y.; Osa, T. Tetrahedron Lett. 1983, 24, 5245-5248; (c) Mulder, A.; Juković, A.; van Leeuwen, F. W. B.; Kooijman, H.; Spek, A. L.; Huskens, J.; Reinhoudt, D. N. Chem. Eur. J. 2004, 10, 11141123; (d) Yamauchi, K.; Takashima, Y.; Hashidzume, A.; Yamaguchi, H.; Harada, A. J. Am. Chem. Soc. 2008, 130, 50245025 .

(5) Lagona, J.; Mukhopadhyay, P.; Chakrabarti, S.; Isaacs, L. Angew. Chem., Int. Ed. 2005, 44, 4844-4870.

(6) Jiao, D.; Biedermann, F.; Scherman, O. A. Org. Lett. 2011, 13 , 3044-3047.

(7) (a) Natansohn, A.; Rochon, P. Chem. Rev. 2002, 102, 41394176; (b) del Barrio, J.; Tejedor, R. M.; Chinelatto, L. S.; Sánchez, C.; Pinol, M.; Oriol, L. Chem. Mat. 2010, 22, 17141723

(8) Urbach, G. Int. Dairy J. 1995, 5, 877-903

(9) Kim, H.-J.; Heo, J.; Jeon, W. S.; Lee, E.; Kim, J.; Sakamoto, S.; Yamaguchi, K.; Kim, K. Angew. Chem., Int. Ed. 2001, 40 1526-1529.

(10) Biedermann, F.; Uzunova, V. D.; Scherman, O. A.; Nau, W. M.; De Simone, A. J. Am. Chem. Soc. 2012, 134, 15318-15323.

(11) del Barrio, J.; Horton, P. N.; Lairez, D.; Lloyd, G. O.; Toprakcioglu, C.; Scherman, O. A. J. Am. Chem. Soc. 2013, 135, $11760-11763$

(12) We note that there could be two possible conformers for $\mathrm{CB}[8] \cdot Z-\mathbf{2}$ and $\mathrm{CB}[8] \cdot Z-\mathbf{1}$ : one where the two cationic groups reside at the same ureidyl carbonyl portal area and another where each of the groups reside at a different $\mathrm{CB}[8]$ portal (see supporting information). For the sake of clarity, only one of the possible conformers is illustrated in Scheme 1 and Figures 1, 3 and 4 .

(13) Kundu, P. K.; Samanta, D.; Leizrowice, R.; Margulis, B.; Zhao, H.; Börner, M.; Udayabhaskararao, T.; Manna, D.; Klajn, R. Nat. Chem. 2015, 7, 646-652.

(14) Appel, E. A.; del Barrio, J.; Dyson, J.; Isaacs, L.; Scherman, O. A. Chem. Sci. 2012, 3, 2278-2281.

(15) Gadde, S.; Batchelor, E. K.; Weiss, J. P.; Ling, Y.; Kaifer, A. E. J. Am. Chem. Soc. 2008, 130, 17114-17119.

(16) Herrmann, A. Angew. Chem., Int. Ed. 2007, 46, 5836-5863.

(17) Velema, W. A.; Szymanski, W.; Feringa, B. L. J. Am. Chem. Soc. 2014, 136, 2178-2191.

(18) Gorostiza, P.; Isacoff, E. Y. Science 2008, 322, 395-399. 
Graphical TOC Entry

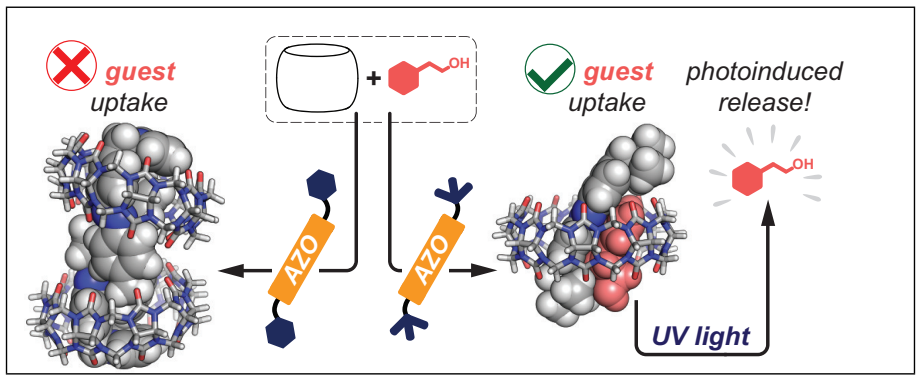

